\title{
Structure of the Human Reovirus Virion at 9.6§ Resolution
}

\author{
Xing Zhang, ${ }^{*}$ Stephen B. Walker, ${ }^{*}$ Max L. Nibert, ${ }^{* *}$ and Timothy S. Baker ${ }^{*}$ \\ *Department of Biological Sciences, Purdue University, West Lafayette, IN 47907 \\ **Department of Microbiology \& Molecular Genetics, Harvard Medical School, Boston, MA 02115
}

Reovirus (family Reoviridae) is a large, icosahedral dsRNA virus with a diameter of $\sim 850 \AA$ and a molecular mass of $129.5 \mathrm{MDa}$.[1] Reovirus virions are composed of eight proteins $(\square 1, \square 2, \square 3, \square 1$, $\square 2, \square 1, \square 2, \square 3$ ) and ten genome segments, which also code for three non-structural proteins ( $\square$ NS, $\square$ NS, and $\square 1 \mathrm{~s}$ ). Six hundred copies each of $\square 3$ and $\square 1$ are organized in the outer capsid in an incomplete, $\mathrm{T}=13 \ell$ icosahedral lattice as $200 \square 1_{3} \square 3_{3}$ heterohexamers. The $\square 3$ and $\square 1$ proteins, which serve viral "protectin" and "penetrin" roles [2], are sequentially degraded by proteolysis inside endo/lysosomes. These events lead to release of the transcriptionally active reovirus core into

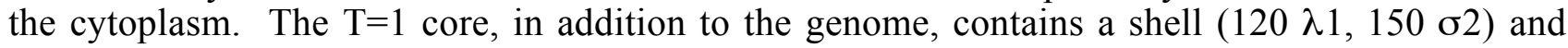
twelve pentameric turrets $(60 \square 2)$ and approximately 12-24 copies of the viral transcriptase $(\square 2, \square 3)$ which produce mRNA transcripts.[3] The $\square 1$ protein, which contains the receptor recognition function and confers tissue tropism, occurs as twelve trimers associated with the $\square 2$ turrets in virions.

Virions (serotype T3D) were embedded in vitreous ice and maintained at $-176^{\circ} \mathrm{C}$ as described.[4] Electron micrographs were recorded under low dose conditions $\left(\sim 24\right.$ electrons $\left./ \AA^{2}\right)$ in a Philips CM200 FEG microscope at a nominal magnification of 38,000 $\square$. Micrographs were digitized with a Zeiss PHODIS scanner with step size of $7 \square \mathrm{m}$ and bin-averaged to give $14 \square \mathrm{m}$ pixels (equivalent to $3.68 \AA$ at the specimen). Twenty-nine micrographs whose defocus ranged from 1.56 to $3.19 \square \mathrm{m}$ underfocus were selected for processing. Particle orientations and origins were determined using a model-based method.[5] The final three-dimensional reconstruction (FIG.1A,B), with corrections made to compensate for the effects of the microscope contrast transfer function, was computed from 3652 particles.[4] The distribution of particle orientations was sufficiently random as measured by the eigenvalue spectrum (all inverse eigenvalues were $<0.01$ ) to allow computation of the reconstruction to the $9.6 \AA$ resolution limit of the data.[4] X-ray crystallographic structures of the core [6] and the $\square 1_{3} \square 3_{3}$ heterohexamer [7] exhibited excellent agreement with the reconstructed density map. The program EMFIT [8] was used to accurately dock various components such as the $\square 1 \square 3$ heterohexamer into the virion map.

Inspection of the density map revealed numerous rod-like features most of which could be ascribed to $\square$-helical secondary structural elements present in the X-ray structures of all five major structural proteins (FIG.1C-E). In addition, novel features present in the reconstructed density but not in the crystal structures were observed. For example, spokes of density emanate from and appear to interconnect the $\square 1$ trimers at sites of local sixfold symmetry in the $T=13$ lattice (FIG.1F). One spoke projects away from each $\square 1$ molecule and merges into an annular ring at the local sixfold axis. Recent evidence for the presence of stabilizing disulfide bonds in the outer capsid of orthoreovirus virions [2,7] is consistent with the observed hub-like structure. [9]

References:

[1] M. L. Nibert. Fields Virology. Third Edition(1996)1557.

[2] A. L. Odegard et al., unpublished.

[3] C. L. Hill et al., Nature Struct. Biol. 6(1999)565.

[4] T. S. Baker et al., Microbiol. Molec. Biol. Reviews 63(1999)862.

[5] T. S. Baker and R. H. Cheng, J. Struct. Biol. 116(1996)120.

[6] K. M. Reinisch et al., Nature 404(2000)960. 
[7] S. Liemann et al., Cell 108(2002)283.

[8] M. G. Rossmann et al., J. Struct. Biol. (in press, 2002).

[9] We thank W. Zhang, C. Xiao, R. Ashmore, A. McGough, R. Bernal, and M. Rossmann for help and discussions, and S. C. Harrison, K. M. Reinisch, and S. Liemann for providing crystal coordinates. Work supported in part by grants from the NIH to TSB (GM33050) and to MLN (AI46440), a shared equipment grant from NSF to TSB (BIR 9112921), and an instrumentation reinvestment grant from Purdue University to the Purdue Structural Biology faculty.

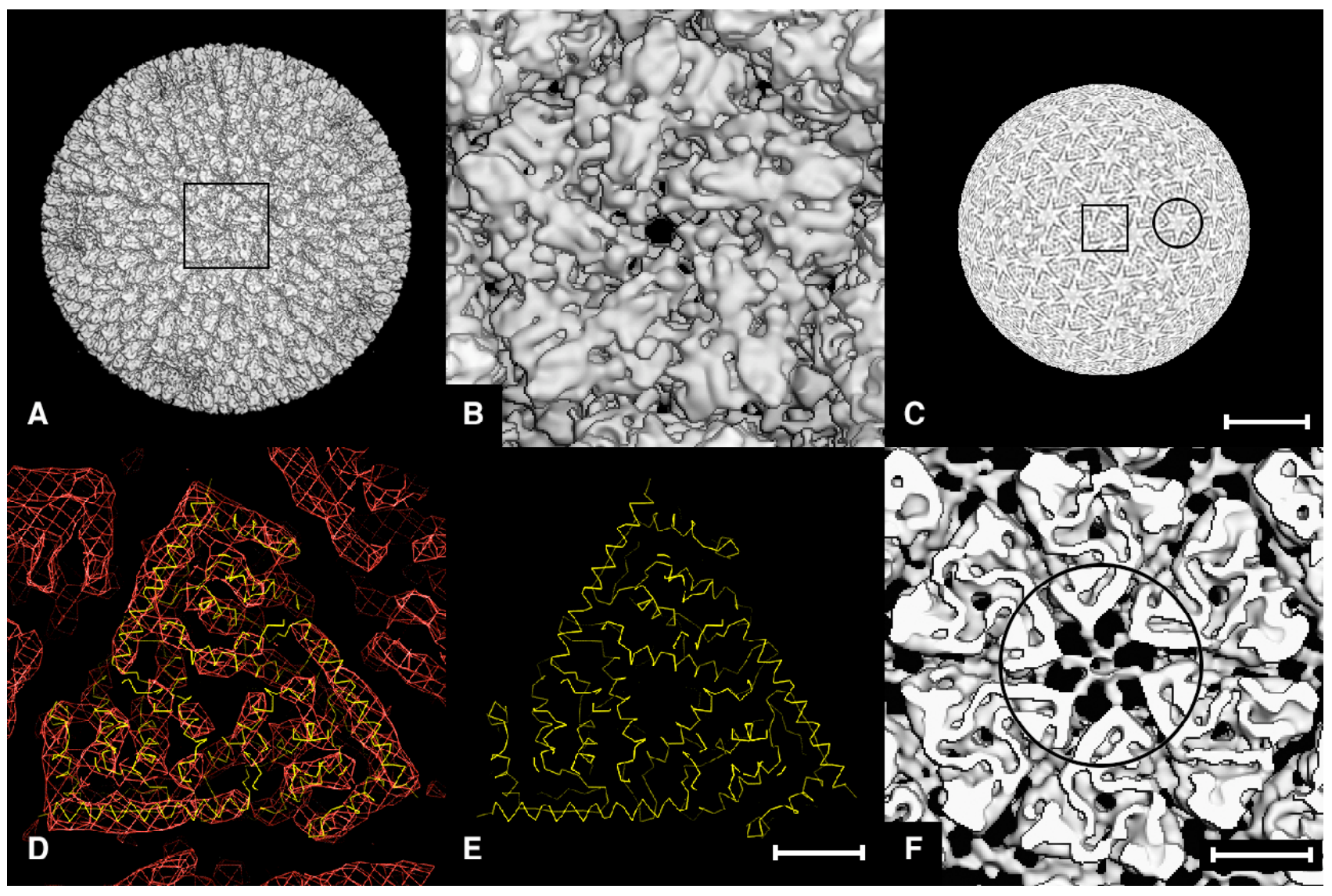

FIG.1. A. Surface representation of the reovirus T3D virion viewed along a 5-fold symmetry axis. One $\square 2$ pentamer (black square) is shown at higher magnification in (B). C. Density projection of the T3D map at a radius of 338 ̊ and viewed along a 3-fold axis (high density appears black). The square demarks a region that includes a small portion of three $\square_{1}$ subunits (enlarged in D). The circle identifies a portion of the P3 channel [1] and six surrounding $\square_{1}$ subunits (also shown in F). D. Fit of the reovirus T1L $\square_{1} \mathrm{X}$-ray crystal structure [7] into the T3D density map. The portion of the map shown, a planar section near the region depicted in $(C$; square box $)$, reveals that several long stretches of $\square$-helices in the $\square_{1}$ trimer fit nicely into rod-like densities in the reconstructed map. Only the $\mathrm{C}_{\square}$ backbone of the $\square_{1} \mathrm{X}$-ray structure is depicted. E. Same as (D) but only showing the X-ray structure. F. Magnified view (shaded surface representation) of a planar section centered about the P3 channel (see encircled region in C). A spoke structure, suspended inside the channel at a particle radius of $\sim 334 \AA$, appears to arise from the association of six density features that project from each of the six $\square_{1}$ subunits that form the channel. Scale bars $=200 \AA(A, C) ; 50 \AA(B, F)$; and $20 \AA$ (D,E). 